\title{
Genistein Inhibited Estradiol-Induced Vascular Endothelial Cell Injury by Downregulating the FAK/Focal Adhesion Pathway
}

\author{
Bing Liu Lili Xub $^{\mathrm{b}} \quad$ Xinming Yu ${ }^{c} \quad$ Xuefei Jiao ${ }^{\mathrm{a}} \quad$ Junwei Yan ${ }^{\mathrm{a}} \quad$ Wei $\mathrm{Li}^{\mathrm{a}}$ \\ Mingjin Guo \\ aDepartment of Vascular Surgery, The Affiliated Hospital of Qingdao University, Shandong, \\ bepartment of Endocrinology and Metabolism, The Affiliated Hospital of Qingdao University, \\ Shandong, 'Department of Vascular Surgery, the Central Hospital of Zibo, Shandong, China
}

\section{Key Words}

Estradiol • Vascular endothelial cells • Genistein • FAK

\begin{abstract}
Background/Aims: In this study, we aimed to investigate the effects of genistein on the focal adhesion signaling pathway through its regulation of FAK. Genistein ultimately restored and alleviated estradiol-induced vascular endothelial injury. Methods: Microarray analysis was used to select differentially expressed genes. MTT assay was performed to detect the cell activity, and ROS test and NO test were performed to detect the degree of damage to HUVECs (human umbilical vein endothelial cells). The relative mRNA expression levels and protein expression levels of FAK were tested by western blot and qRT-PCR. GO functional analysis and KEGG pathway analysis were applied to predict the possible relationship between functions and related pathways, and transwell assay was used to detect cell invasion and migration. Results: FAK was highly expressed in the HUVECs treated with estradiol (HU-ESTs). Cell viability and NO level decreased, whereas ROS level increased in the HU-ESTs. Effective knockdown of FAK in HU-ESTs elevated cell viability and NO levels while suppressing ROS levels. In addition, inhibition of $F A K$ greatly decreased cell invasion and migration, while the overexpression of $F A K$ enhanced cell invasion and migration. KEGG further indicated focal adhesion pathways were activated. Genistein elevated HU-EST viability, and NO and ROS level increased in a concentration dependent manner. Transwell and western blot assays revealed that genistein could reduce the FAK expression levels and alleviate the damage to the HU-ESTs. Conclusion: FAK overexpression promoted invasion and migration of the HU-ESTs. However, genistein greatly suppressed $F A K$ and estradiol-induced vascular endothelial cell injury.

B. Liu and L. Xu contributed equally to this work. 


\section{Cellular Physiology Cell Physiol Biochem 2018;49:2277-2292 and Biochemistry \begin{tabular}{c|c} 
DOI: 10.1159/000493830 \\
Published online; 27 September 2018
\end{tabular} $\begin{aligned} & \text { O } 2018 \text { The Author(s). Published by S. Karger AG, Basel } \\
& \text { www.karger.com/cpb }\end{aligned}$}

Liu et al.: Genistein Inhibited Estradiol-Induced Vascular Endothelial Cell Injury

\section{Introduction}

Cardiovascular disease is increasingly regarded as one of the most serious diseases, with high mortality and morbidity worldwide [1]. Vascular endothelial injury and dysfunction play an important role in causing cardiovascular disease. Vascular endothelial injury may to varying degrees reduce or damage endothelial cell coagulation and anticoagulant function, leading to a hypercoagulable state of the blood, which easily induces thromboembolic disease, and it is the first step in the development of cardiovascular disease [2]. It has been reported that the development of vascular endothelial injury accompanied with hypertension is a contributing factor in the development of atherosclerosis and thrombosis [2]. Additionally, in recent years, the study found that vascular complications, such as vascular endothelial dysfunction and vascular inflammation, could induce changes in arterial structural to some extent. In addition, diabetes and peripheral arterial disease are usually induced by vascular complications as well [3]. Thus, it is critical to protect vascular endothelial cells to prevent the occurrence and progression of cardiovascular disease and thromboembolic disease. It is also necessary to study the mechanism of vascular endothelial cell injury.

Genistein is a soy product with high concentrations of phytoestrogens [4]. Genistein can be used as a dietary supplement because it has estrogen-like activity, and researchers have explored its biological role in diseases such as cancer and inflammation [5]. Researchers have explored its positive biological role in protecting vascular endothelial cells and reducing inflammation. Du DS et al. found that genistein could protect gastric vascular endothelial cells by stimulating the PI3K/Akt signaling pathway to prevent gastric ischemia-reperfusion injury in a capsaicin receptor-mediated manner [6]. Sang Hun Lee et al. suggested that genistein could enhance the biological activity of endothelial cell colony forming cells by stimulating the expression of the estrogen receptor (ER) and promote cardiac regeneration [7]. However, the role and potential mechanism of genistein in estradiol-induced endothelial injury is still unknown.

Focal adhesions (FAs) are the mechanical connection of the actin cytoskeleton to the extracellular matrix. FAs serve as a signal transduction platform that can aggregate large amounts of signaling molecules and complexes [8]. FAK is an important intracellular tyrosine kinase that can participate in a variety of cellular activities such as cell migration and invasion [9]. It can be combined with the whole protein receptor and play an indispensable role in integrin-mediated signal transduction [8]. Numerous studies have shown that the expression of $F A K$ was significantly increased in many malignancies and was associated with poor prognosis of patients with cancers [10]. In this study, we mainly discussed the function of $F A K$ in cells and revealed that the focal adhesion signaling pathway could be activated when $F A K$ was overexpressed, which eventually has an adverse effect on the activity of vascular endothelial cells.

We hypothesize that genistein could regulate estradiol-induced cardiovascular endothelial cell injuries. In addition, the damage of focal adhesions to vascular endothelial cells could not be ignored. Consequently, we speculated that the network between genistein, FAK and focal adhesion signaling pathways might have some effects on estradiol-induced cardiovascular endothelial cell injuries. This study was developed based on three aspects: whether the HU-ESTs (human umbilical vein endothelial cells exposed to estradiol) could cause endothelial cell injuries; how FAK would function in the HU-ESTs; and whether genistein could alleviate endothelial cell injuries via the FAK signaling pathway. Hopefully, this study provides new treatment options and inspires additional scientific research. 


\section{Cellular Physiology Cell Physiol Biochem 2018;49:2277-2292 and Biochemistry \begin{tabular}{l|l} 
DOI: 10.1159/000493830 & (c) 2018 The Author(s). Published by S. Karger AG, Basel \\
www.karger.com/cpb
\end{tabular}

\section{Materials and Methods}

\section{Cell culture and treatments}

Human umbilical vein endothelial cells (HUVECs) were purchased from BeNa Culture Collection (http://www.bnbio.com) and cultured in 90\% F-12K Medium, 10\% FBS (Invitrogen, Carlsbad, CA, USA) supplemented with $0.1 \mathrm{mg} / \mathrm{mL}$ heparin and $0.03 \mathrm{mg} / \mathrm{mL}$ ECGS (Endothelial Cell Growth supplements) mix. Cells were cultured in a humidified incubator at $37^{\circ} \mathrm{C}$ in $5 \% \mathrm{CO}_{2}$. Cells were exposed to estradiol (Sigma, Alcobendas, Spain) by serial dilutions of a stock solution. Control cells were exposed to the same vehicle $(0.1 \%$ ethanol) or ICI182780 ( $0.1 \%$ DMSO). All treatments were added in hormone free medium, and the experiments were performed at $75-80 \%$ of confluence.

\section{Cell transfection}

pcDNA3.1, pcDNA3.1-FAK, si-NC (transfected with negative control), and si-FAK were purchased from BeNa Culture Collection (Qiagen, Valencia, CA, USA). For the gain-of-function analyses, HUVECs were seeded into each well of 6-well plate and cultured in F-12K medium containing 10\% FBS; then, pcDNA3.1, pcDNA3.1-FAK, si-NC and si-FAK were transfected into the HUVECs using Lipofectamine-2000 (Invitrogen) according to the manufacturer's protocol. After 48 hours, the transfected cells were harvested for subsequent experiments. The methods used to analyze transfection efficacy include qRT-PCR and western blot. Groups were set as follows: (1) control group, (2) pcDNA3.1 group, (3) pcDNA3.1-FAK group, (4) si-NC group and (5) si-FAK group.

\section{Microarray analysis}

The mRNA microarray expression data have been deposited in the National Center for Biotechnology Information's (NCBIs) Gene Expression Omnibus with accession numbers GSE 16683, which contains three HUVEC samples and three estradiol-induced HUVEC samples. Differentially expressed mRNA were screened with $P$-value $<0.05$ accompanied with $\log _{2}$ (fold change) $>1$. The results were visualized through heatmap analysis.

\section{GO analysis and KEGG analysis}

The total standardized mRNA expression data were uploaded to GSEA v3.0 software. Genome enrichment analysis was performed by selecting KEGG pathway genome and GO genome. Using the default weighted enrichment statistics and the processing data 1000 times, normalized $P<0.05$ was considered to be significant. Next, the highest and lowest 7 results in the GSEA report were selected and used in the "ggplot2" package in the R language for graphical processing. Additionally, we used the R language "GSEA Base" package to process the data. We retained more than 10 genomes with less than 500 genes. $P$ value was adjusted by the Benjamini-Hochberg method. Using 1000 replacement tests to identify the significantly different pathways and GO items, the $\mathrm{p}$ value was less than 0.05 .

\section{Real-time quantitative PCR ( $q R T-P C R$ ) analysis}

Using TRIzol to extract total RNA, cDNA was synthesized using m-mlv reverse transcriptase (BioTeke, Beijing, China). Power SYBR Green PCR Master Mix (Applied Biosystems) and ABI 7500 real-time PCR system (Applied Biosystems) were used to amplify the cDNA fragments. Primers used in this study were shown in Table 1. Samples were analyzed in triplicate, and the housekeeping gene glyceraldehyde-3-phosphate dehydrogenase (GAPDH) served as an endogenous control.

Western blot analysis

HUVECs were treated with or without estradiol at a fixed time after preincubation with fixed concentrations. Cells were harvested and lysed by scraping in ice-cold
Table 1. Primer Sequences for qRT-PCR

\begin{tabular}{lc}
\hline Genes & Sequences \\
\hline FAK & F: 5'-GCTCCCTTGCATCTTCCAGT-3' \\
& R: 5'-ATTGCAGCCCTTGTCCGTTA-3' \\
GAPDH & F: 5'-GGAAAGCTGTGGCGTGAT-3' \\
& R: 5'-AAGGTGGAAGAATGGGAGTT-3'
\end{tabular}




\section{Cellular Physiology Cell Physiol Biochem 2018;49:2277-2292 \begin{tabular}{l|l|l} 
and Biochemistry & Dublished online: 27 September 2018 & $\begin{array}{l}\text { O } 2018 \text { The Author(s). Published by S. Karger AG, Basel } \\
\text { www.karger.com/cpb }\end{array}$ \\
\cline { 2 - 3 }
\end{tabular}}

RIPA buffer (Santa Cruz, CA, USA). Protein concentrations were determined by BCA assay (Solarbio, Beijing, China), and total protein for each $50 \mu \mathrm{g}$ sample was resolved on polyacrylamide gel and blotted onto PVDF membranes for immunoblotting. The membranes were incubated with $5 \%$ nonfat dry milk in TBST for 1 $\mathrm{h}$ at room temperature. The primary antibodies were incubated with the membranes, including anti-FAK (1:1000), anti-FLNA (1:250000), anti-ZYX (1:10000), anti-BCAR1 (1:1000), anti-SHC1 (1:5000) and antiGAPDH (1:5000) (Abcam, Cambridge, MA, USA), and then incubated at $4{ }^{\circ} \mathrm{C}$ overnight. After incubation, the membranes were washed, incubated with horseradish-peroxidase secondary antibody IgG (1:5000) for $1 \mathrm{~h}$, and washed again. Protein band densities were quantified using an enhanced chemiluminescence detection system (syngene, USA), and the protein levels were analyzed using ImageJ software.

\section{MTT assay}

The HUVECs were seeded into 96 wells with $1 \times 10^{4}$ cells/well density. To measure the cell viability of HUVECs and HU-ESTs, cells were grown to $95 \%$ confluence, while measuring the cell viability of cells after treatment with genistein, cells were grown to $80 \%$ confluence. Cells were exposed for 24, 48, and $72 \mathrm{~h}$. After treatment, $20 \mu \mathrm{L}$ of MTT was added to a final $0.5 \mathrm{mg} / \mathrm{mL}$ concentration in each well, and the cells were incubated at $37^{\circ} \mathrm{C}$ for $4 \mathrm{~h}$. Then, $150 \mu \mathrm{L}$ of dimethyl sulfoxide (DMSO) was added, and the optical density was measured at $490 \mathrm{~nm}$ using the Tecan Infinite F200 (Switzerland).

\section{Cell invasion and migration assay}

For transwell migration analysis, cells $\left(4 \times 10^{5}\right)$ and uncoated membranes (24 well pore inserts; Aperture, 8 microns; BD Biosciences, San Jose, CA, USA) were placed in the upper chamber. For analyzing the invasion rate, the matrix (BD bioscience) was inserted into wells and incubated at $37^{\circ} \mathrm{C}$ for $45 \mathrm{~min}$. In both measures, cells were placed in the upper chamber without serum. Then, 20\% FBS (GIBCO BRL, Grand Island, NY, USA) was used to fill the lower compartment as a chemical attractant. Cells were incubated for $24 \mathrm{~h}$, and immigrated or invasive cells were removed through a cotton swab. The cells were transferred to the surface of the film and stained with $0.1 \%$ crystal violet stain. Cell counting was performed using light microscopy and was performed three times to obtain an average value.

\section{Measuring NO level}

Nitrite and nitrate levels of hydrolytic cells were generally considered to be the indicators of NO. The levels of nitrite and nitrates were determined using the manufacturer's recommendations. In brief, a series of nitrite concentrations was used to prepare the standard curve. Then, $100 \mu \mathrm{l}$ samples were added into the 96-well microporous plate, and Griess reagents I and II were added to the plate and mixed thoroughly. Finally, the optical density value (OD) was measured at $550 \mathrm{~nm}$ with the spectrophotometer (Bio-Rad Laboratories).

\section{Measuring ROS level}

According to the manufacturer's instructions, the intracellular ROS levels were measured using 2, 7-dichlorofluorescein diacetate (dcfh-da) (peroxide sensitive fluorescent probe). The cells were harvested after washing with PBS buffer and then cultured in serum-free DMEM medium (incubated for 30 min at $37^{\circ} \mathrm{C}$ ). The fluorescence intensity of DCF was evaluated by fluorescence microscopy, and the fluorescence intensity of DCF was quantitatively measured using Image Software.

\section{Statistical analysis}

Data were presented as the means \pm standard error (SD) from three independent experiments. Group differences were analyzed by Student's t-test or one-way ANOVA using GraphPad Prism 6 software (La Jolla, CA, USA). $P<0.05$ was considered statistically significant. 


\section{Results}

The expression of FAK was enhanced in estradiol-treated HUVECS

Based on the Affymetrix Human Genome U133 Plus 2.0 Array GSE16683, the top 10 highest expressing mRNAs and the 10 lowest expressing mRNAs on the heatmap were screened; FAK had higher expression in the HU-EST group compared with the HUVEC group (Fig. 1A, ${ }^{*} P<0.05, \mid \log _{2}$ (Fold Change) $\mid>1$ ). To identify the levels of $F A K$ in damaged vascular endothelial cell, we used estradiol to establish a model of vascular endothelial cell injury, referred to as HU-EST. The MTT method demonstrated that cell viability was decreased when

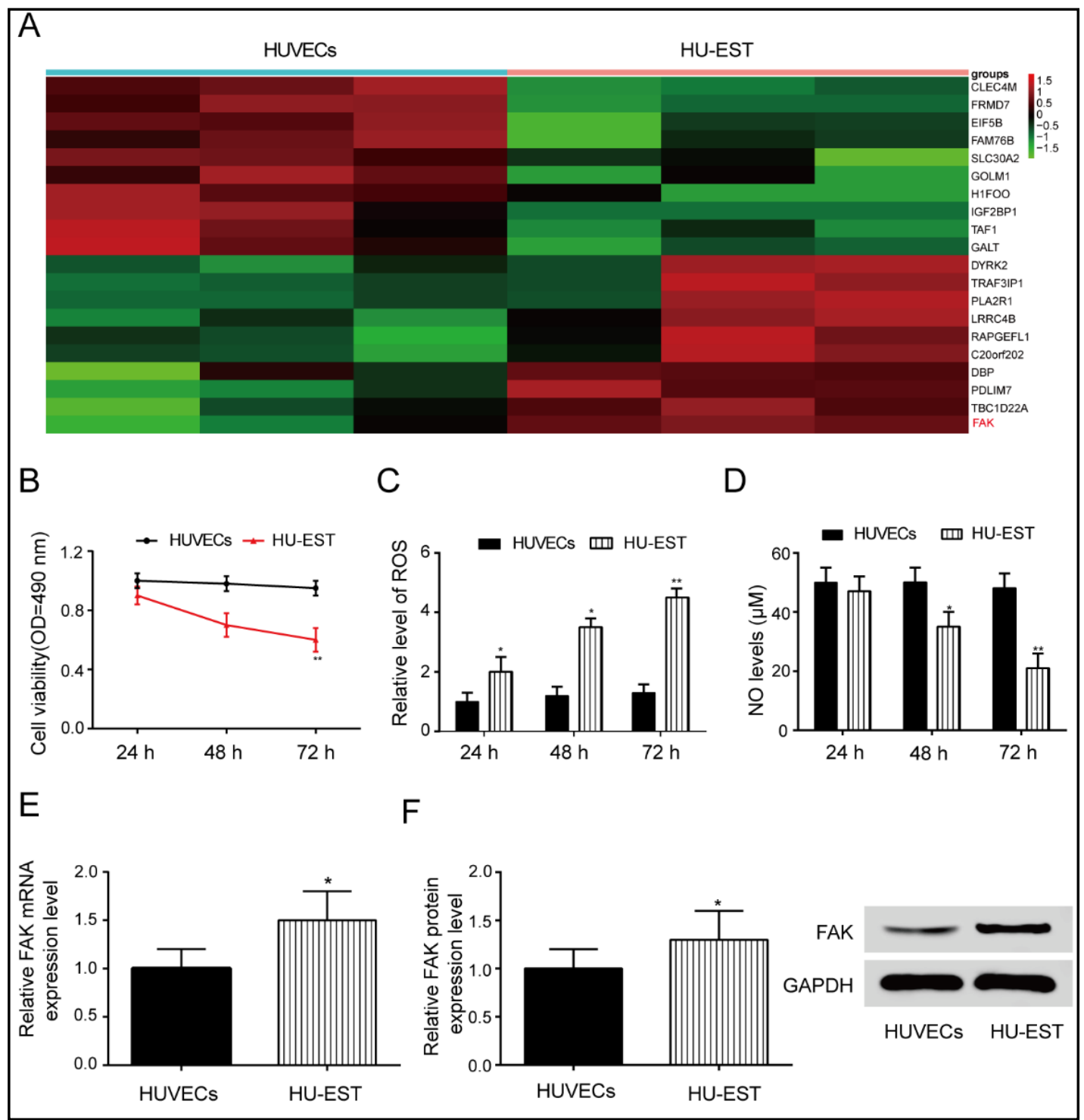

Fig. 1. The expression of FAK was increased in the estradiol-treated HUVECs. (A) Heatmaps showed the top 20 most significantly differentially-expressed genes between estradiol treated HUVECs (HU-ESTs) and normal control (HUVECs). (B) MTT was used to detect cell viability in the HUVEC group and HU-EST group. (C) The relative level of ROS was examined by ROS test in the HUVEC group and HU-EST group. (D) The NO level was examined by NO test in the HUVEC group and HU-EST group. (E) The relative FAK mRNA expression level was tested by qRT-PCR in the HUVEC group and HU-EST group. (F) FAK protein expression level was examined by western blot in the HUVEC group and HU-EST group. ${ }^{*} \mathrm{P}<0.05,{ }^{* *} \mathrm{P}<0.01$ indicated statistical significance compared with the HUVEC group.

\section{KARGER}




\section{Cellular Physiology Cell Physiol Biochem 2018;49:2277-2292 and Biochemistry \begin{tabular}{l|l} 
DOI: 10.1159/000493830 & $\begin{array}{l}\text { O 2018 The Author(s). Published by S. Karger AG, Basel } \\
\text { www.karger.com/cpb }\end{array}$
\end{tabular} \\ Liu et al.: Genistein Inhibited Estradiol-Induced Vascular Endothelial Cell Injury}

HUVECs were treated by estradiol, especially at $72 \mathrm{~h}\left(\mathrm{Fig} .1 \mathrm{~B},{ }^{* *} \mathrm{P}<0.01\right)$. ROS testing showed that ROS levels in the HU-EST group were increased over time, especially at $72 \mathrm{~h}$ (Fig. 1C, ${ }^{*} P<0.05,{ }^{* *} P<0.01$ ). In contrast, NO levels in the HU-EST group continued to decline, with the most pronounced decline at $72 \mathrm{~h}$ (Fig. $1 \mathrm{D},{ }^{*} P<0.05,{ }^{* *} P<0.01$ ). According to qRT-PCR and western blot analyses, mRNA and protein levels of FAK were higher in HU-ESTs compared with HUVECs (Fig. 1E-1F, ${ }^{*} P<0.05$ ). These results not only indicated that a model of vascular endothelial cell injury was developed but also showed that $F A K$ was highly expressed in the HU-EST group.

\section{FAK promoted the invasion and migration of HU-ESTS}

GO term enrichment analysis varied from GO classification as well as the expression fluctuation of GEGs. GO analysis revealed that some functions related to cell movement were activated (Fig. 2A-2B), such as actin cytoskeleton and regulation of body fluid level.

In addition, Fig. 3A shows seven of the highest scores of GO functional analysis for HUVEC and HU- EST, according to an enrichment score reported by GSEA. FAK was transfected into HU-ESTs, and qRT-PCR and western blot were used to measure the transfection efficiency (Fig. 3B, ${ }^{*} P<0.05$ ). MTT experiments showed that cell activity continued to increase in the siFAK group, especially at $72 \mathrm{~h}$. In contrast, cell activity was obviously reduced in the pcDNA3.1FAK group over time compared with the pcDNA3.1 group (Fig. $3 \mathrm{C},{ }^{*} \mathrm{P}<0.05$ ). Next, the relative levels of ROS were significantly increased, but the NO level was obviously decreased in the pcDNA3.1-FAK group compared with the pcDNA3.1 group, while the opposite result appeared in the si-FAK group (Fig. 3D-3E, ${ }^{*} P<0.05$ ).

Migration and invasion experiments revealed that the number of migratory and invasive cells was remarkably enhanced when $F A K$ was overexpressed but decreased when $F A K$ was knocked down (Fig. 4A-4D, ${ }^{*} P<0.05$ ). In summary, FAK damaged vascular endothelial cells and promoted the migration and invasion of the HU-ESTs.

\section{FAK could activate the focal adhesion pathway}

KEGG analysis was performed to determine the pathways involved in the processes occurring in HUVECs treated with estradiol. From the results in Fig. 5A and Fig. 5B, we found that the focal adhesion pathway was activated.

In addition, according to the enrichment score reported by GSEA, Fig. 6A shows seven of the highest scoring pathways in the HUVECs and HU-ESTs. After cross-examination of these effects, we focused on the selective focal adhesion signaling pathway activated in the HUESTs (Fig. 6B). Therefore, we speculated that the focal adhesion pathway may affect some gene functions, thus affecting the HU-ESTs. We observed the downstream target proteins of FAK (BCAR1 and SHC1) in the focal adhesion pathway (Fig. 6C).

Based on Affymetrix Human Genome U133 Plus 2.0 Array GSE16683, we investigated the 3 genes (FAK, FLNA and ZYX) in the focal adhesion pathway that had significantly higher expression in estradiol-treated HUVECs $(n=3)$ compared with untreated HUVECs $(n=3)$ (Fig. 6D, $P<0.05, \log _{2}$ (Fold Change) $>1$ ). To verify that FAK could activate the focal adhesion pathway, western blot was used to measure the expression of FLAN, ZYX, BCAR1 and SHC1 proteins (Fig. 6E). The results suggested that $F A K$ stimulated proteins related to the focal adhesion pathway and thus activated the focal adhesion pathway.

\section{Genistein reduced the FAK expression level and alleviated the damage to HU-ESTs}

The STITCH interaction network found that FAK was associated with genistein (Fig. 7A). To verify the appropriate concentrations were used in the experiment, various concentrations of genistein $(10,50$ and $100 \mu \mathrm{M})$ were applied. As expected, some concentrations of genistein failed to impact the cell viability and levels of ROS and NO in the HUVECs (Fig. 7B-7D). Next, we further investigated the effects of various concentrations of genistein on the HU-ESTs (10, 50 and $100 \mu \mathrm{M}$ ). The results varied considerably at different concentrations to counteract the changes induced by estradiol, suggesting that genistein protected the HUVECs against the injury induced by estradiol in a concentration dependent manner (Fig. 7E-7G, ${ }^{*} P<0.05$, 


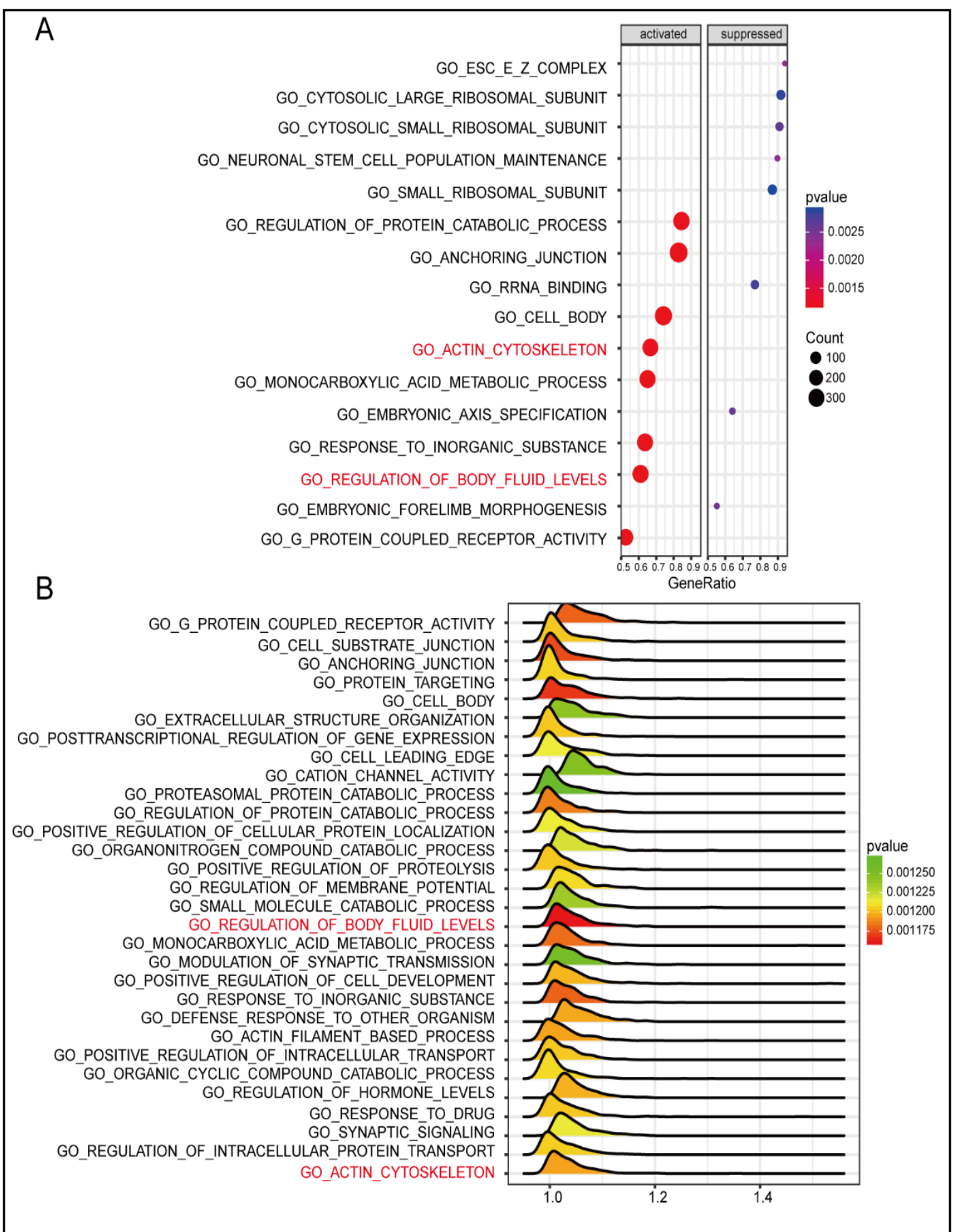

Fig. 2. Functional annotations for the upregulated genes in the HU-ESTs. (A and B) Dotplot and Joyplot suggested the distributions of some GO term gene sets in all the differentially expressed genes.

${ }^{* *} P<0.01$ ). To ensure the reproducibility and simplicity of the experiment, the concentration of $100 \mu \mathrm{M}$ genistein was selected as the fixed concentration for subsequent experiments. Then, qRT-PCR and western blot experiments were performed and confirmed that the expression of $F A K$ was significantly decreased when $100 \mu \mathrm{M}$ genistein was added to the HU-ESTs.

However, when we overexpressed $F A K$ in GEN+FAK group, the expression of $F A K$ was increased, and there was no notable difference compared to the NC group (Fig. 8A-8B, ${ }^{*} P<0.05$ ). MTT, ROS and NO tests were performed, and the experimental results were as follows. MTT KARGER 


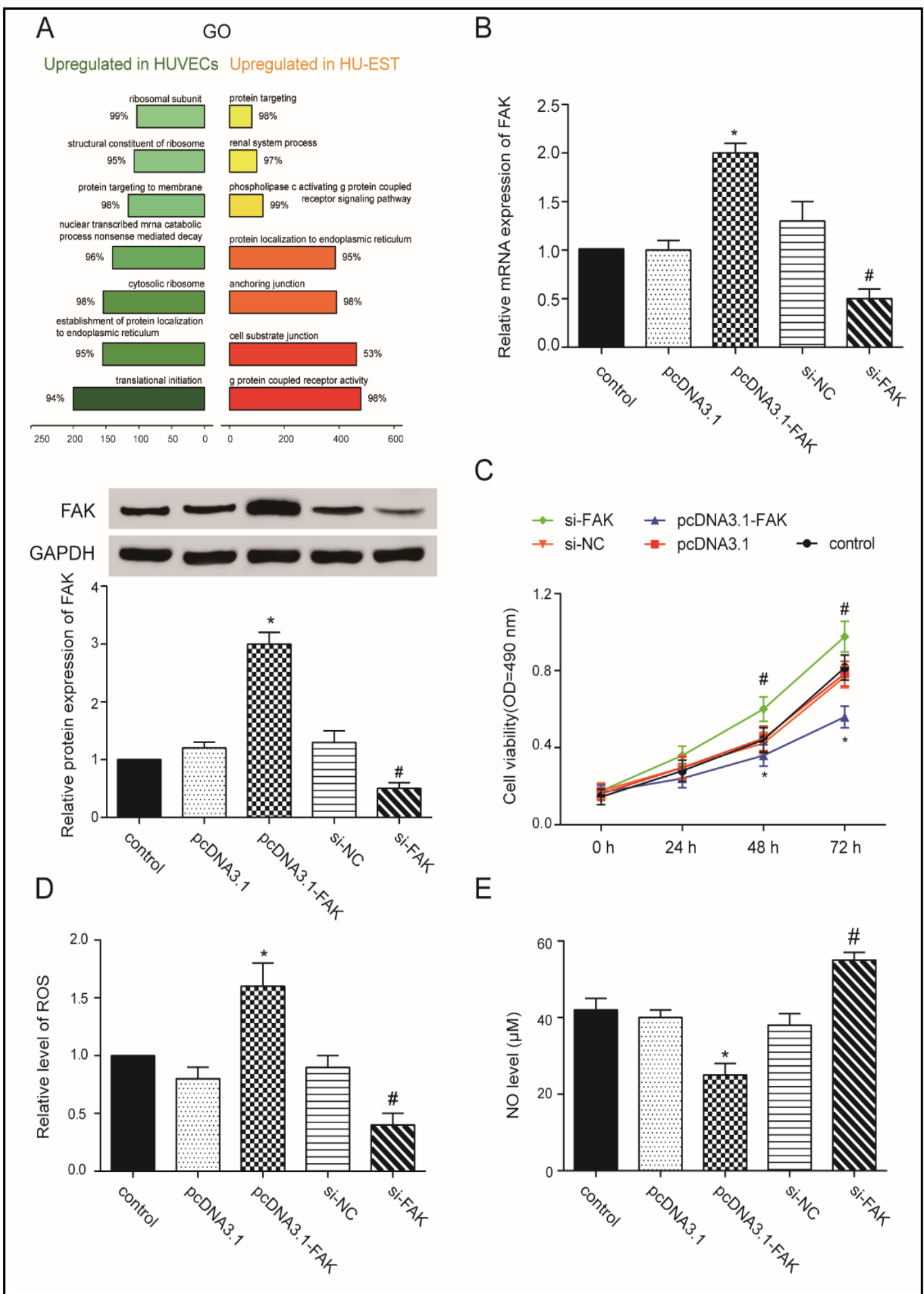

Fig. 3. FAK overexpression inhibited the HU-EST activity and intensified the HU-EST injury. (A) Plot of the seven most significantly enriched GO terms activated in HUVECs or HU-ESTs. In B-E, the HU-ESTs had specific treatments. (B) qRT-PCR and western blot tested the expression of FAK. (C) MTT was applied to test the cell viability. (D) The relative level of ROS was examined. (E) NO levels were examined. ${ }^{*} \mathrm{P}<0.05$ indicated statistical significance compared with the pcDNA3.1 group. $\# \mathrm{P}<0.05$ indicated statistical significance compared with the si-NC group. 


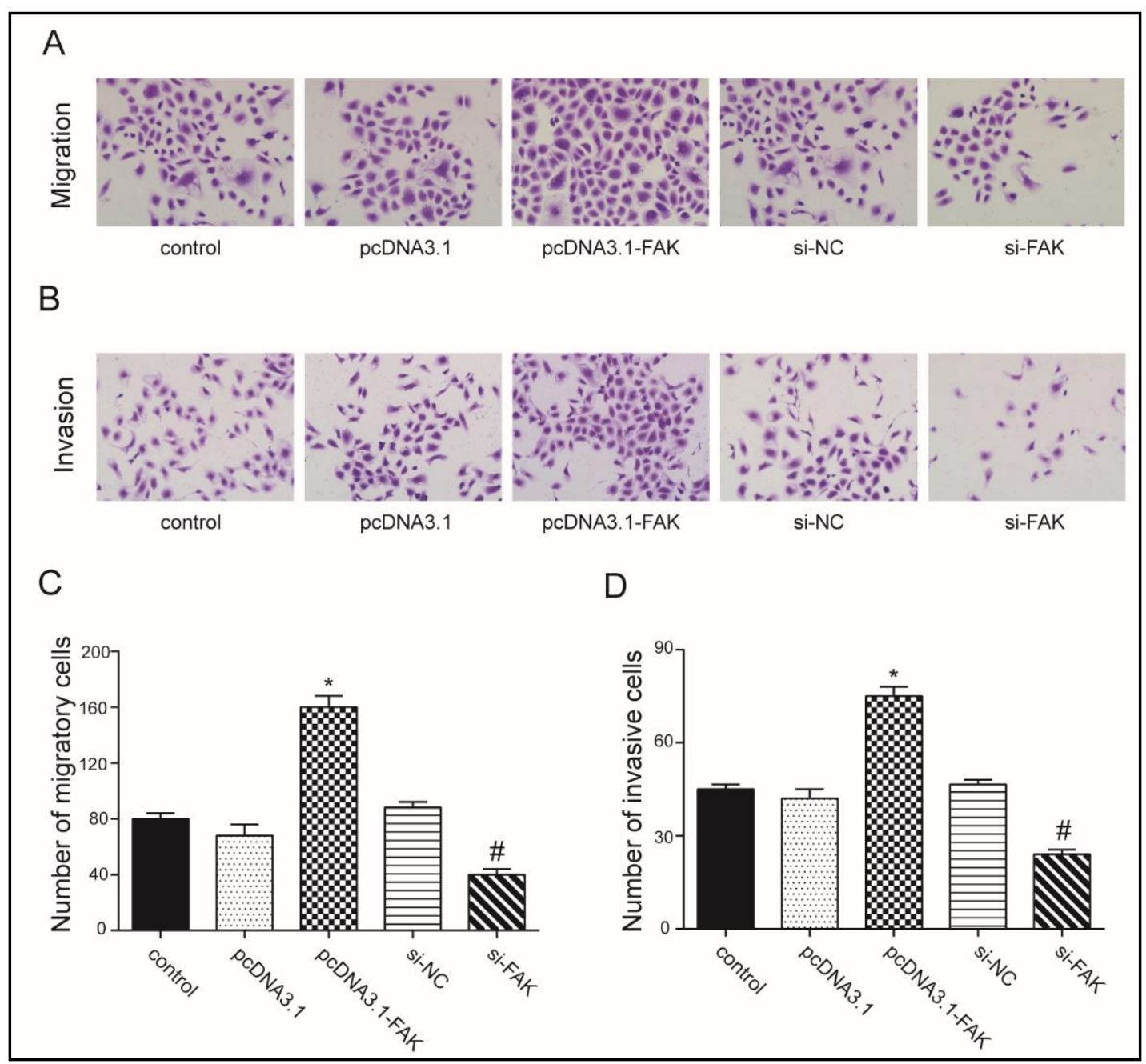

Fig. 4. FAK promoted the migration and invasion of the HU-ESTs. In A-D, HU-ESTs were treated with specific treatments. (A) Transwell experiments were conducted to detect migratory ability. (B) Transwell experiments were conducted to detect invasive ability. (C) The histogram shows the number of migrating cells. (D) The histogram shows the number of invasive cells. ${ }^{*} \mathrm{P}<0.05$ indicated statistical significance compared with the pcDNA3.1 group. $\# \mathrm{P}<0.05$ indicated statistical significance compared with the si-NC group.

and NO tests showed that cell activity and NO level of the GEN group was obviously higher than that of the NC group; however, in the GEN+FAK group, cell activity and NO level were lower, and the same results were found in the NC group (Fig. 8C, $8 \mathrm{E},{ }^{*} P<0.05$ ). In contrast, ROS test results showed that when $100 \mu \mathrm{M}$ genistein was added to the HU-ESTs, the relative levels of ROS were significantly reduced. However, after FAK was overexpressed, the relative levels of ROS were restored, with no significant difference from that in the NC group (Fig. 8D, ${ }^{*} P<0.05$ ). Transwell assay was also used to test the migration and invasion abilities in each group. Similar results were observed; migratory and invasive cells significantly decreased in the presence of genistein, but migration and invasion recovered in the GEN $+F A K$ group (Fig. 8F-8J, ${ }^{*}<<0.05$ ). In addition, we performed western blot experiments to determine the expressions of FLAN, ZYX, BCAR1 and SHC1. The results showed genistein could downregulate the expression of genes involving in the focal adhesion pathway, thus suppressing the focal adhesion pathway (Fig. 8K). These experiments showed that genistein could reduce the expression level of $F A K$, which could suppress the focal adhesion pathway and ultimately moderate the degree of damage to HUVECs induced by estradiol.

\section{KARGER}


A

B
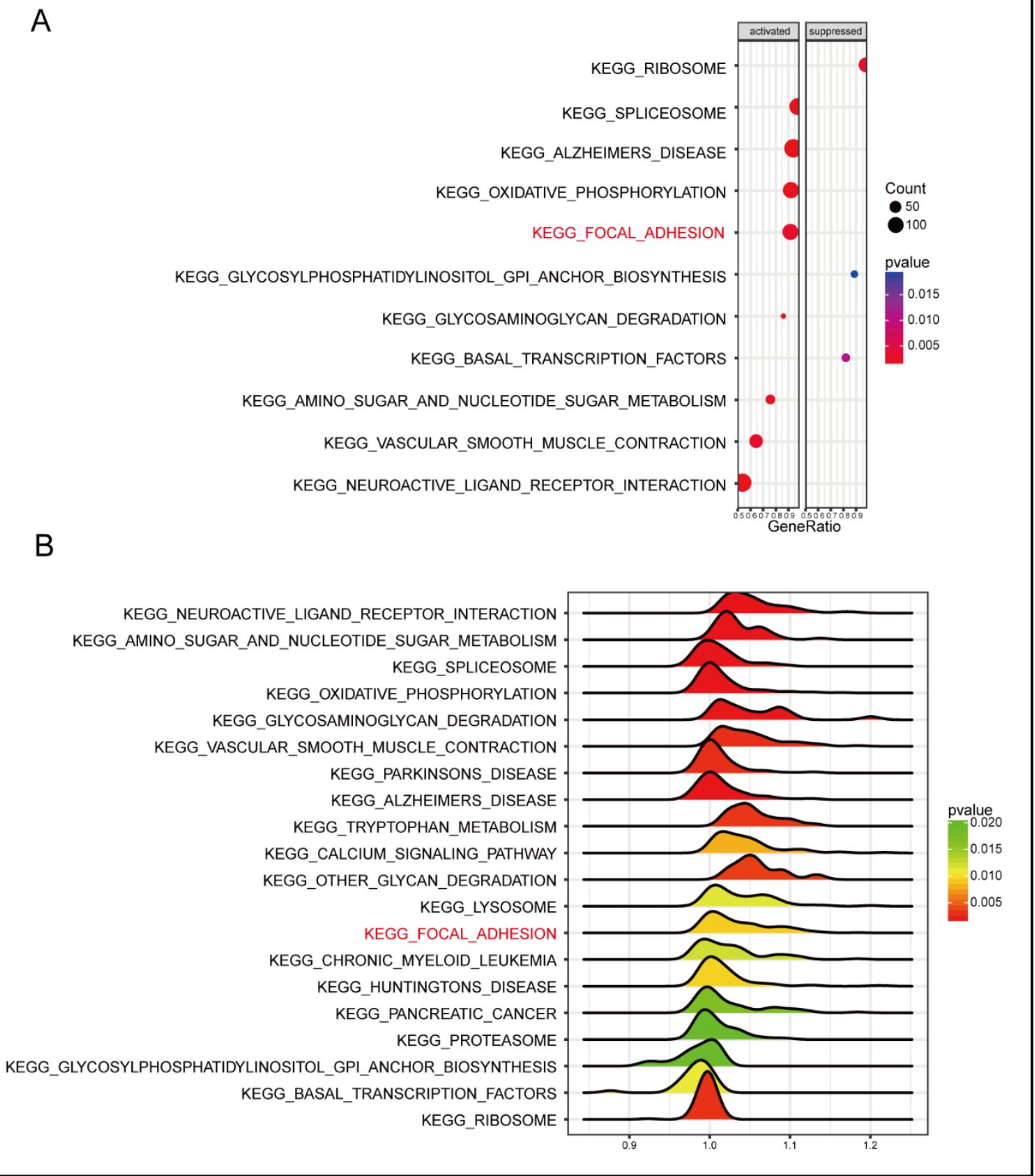

Fig. 5. KEGG annotations for upregulated genes in the HU-ESTs. (A and B) Dotplot and Joyplot suggested the distributions of some KEGG pathway gene sets in all the differentially expressed genes. 


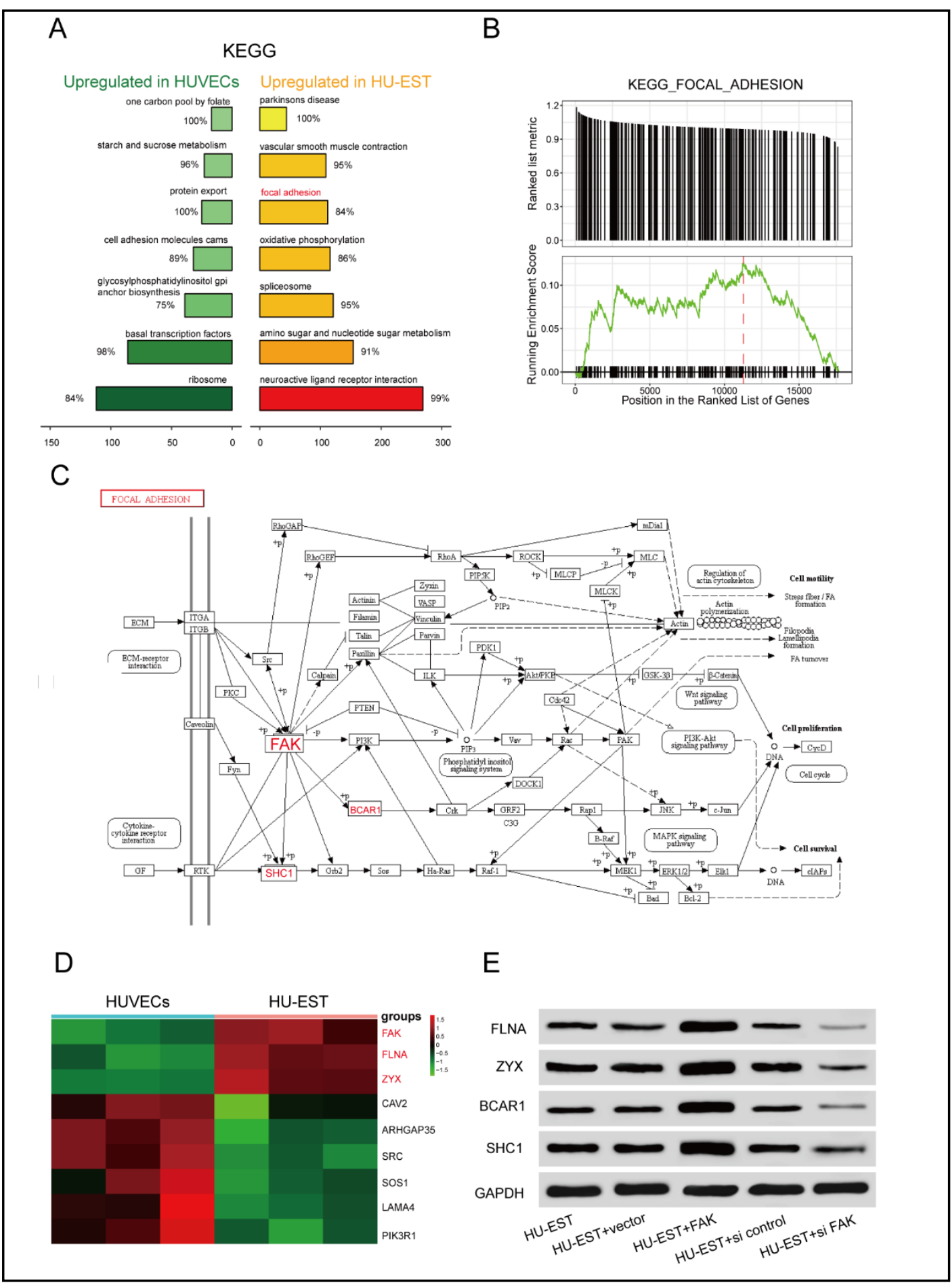

Fig. 6. FAK activated the focal adhesion pathway. (A) Plot of the seven most enriched KEGG pathways in the HUVECs and HU-ESTs. Pathways were ordered by normalized enrichment score (NES). Percentage beside the bar indicated the proportion of differential genes in a pathway gene set. (B) GSEA plot showed that most genes in the KEGG focal adhesion pathway were discovered in the region where the genes are overexpressed in the HU-ESTs. (C) The focal adhesion signal pathway was downloaded from the KEGG website and shows the downstream target genes of FAK. (D) Heatmaps show 9 differentially expressed genes in the focal adhesion pathway. (E) Western blotting examined the expression levels of FLAN, ZYX, BCAR1 and SHC1 proteins. 


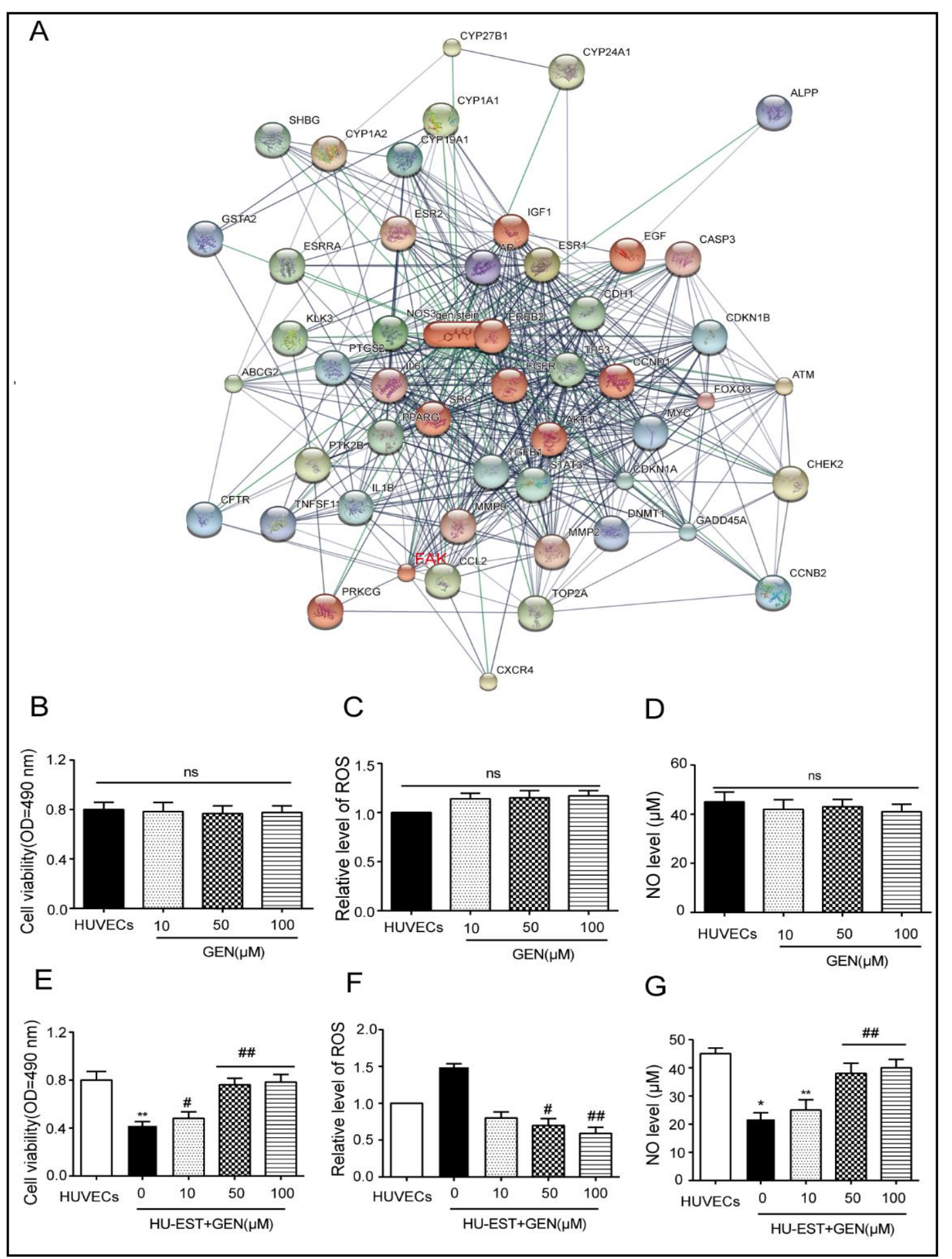

Fig. 7. Genistein reduced the FAK expression level and alleviated the damage to the HU-EST cells. (A) STITCH predicted an interaction between genistein and FAK. In B-D, the GEN group represented HUVECs treated with $10 \mu \mathrm{M}, 50 \mu \mathrm{M}$ and $100 \mu \mathrm{M}$, respectively. (B) MTT was used to detect cell viability. (C) ROS test was used to detect relative levels of ROS. (D) NO test was used to detect NO level. In E-G, HU-EST+GEN group represented HU-ESTs treated with $0 \mu \mathrm{M}, 10 \mu \mathrm{M}, 50 \mu \mathrm{M}$ and $100 \mu \mathrm{M}$, respectively. (E) MTT was used to detect cell activity. (F) ROS test was used to detect relative levels of ROS. (G) NO test was used to detect NO level. ${ }^{*} \mathrm{P}<0.05$. ${ }^{* *} \mathrm{P}<0.01$ indicated statistical significance compared with HUVEC group. $\# \mathrm{P}<0.05, \# \# \mathrm{P}<0.01$ indicated statistical significance compared with the HU-EST group. 
A
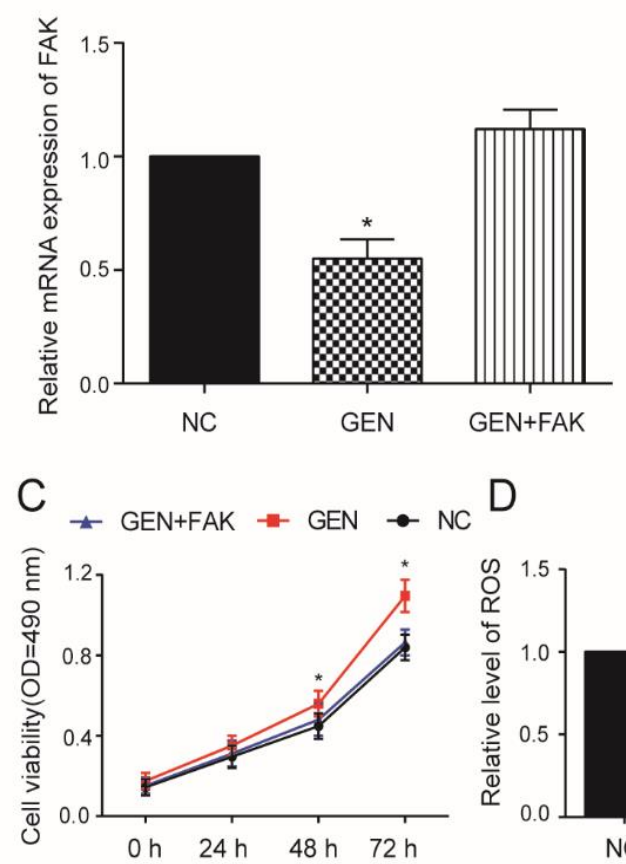

$\mathrm{D}$

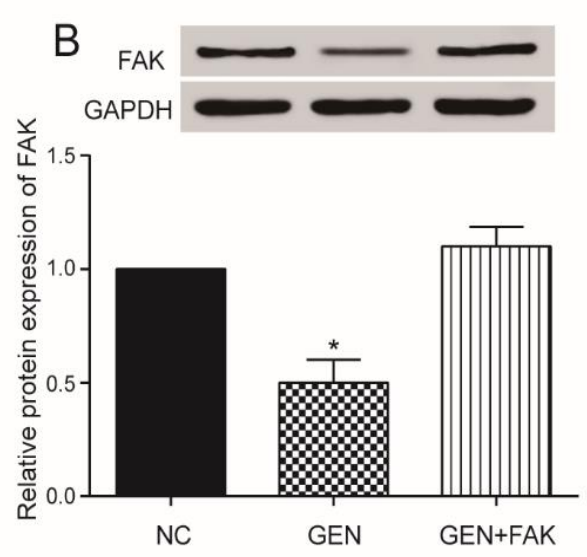

E

$\mathrm{F}$
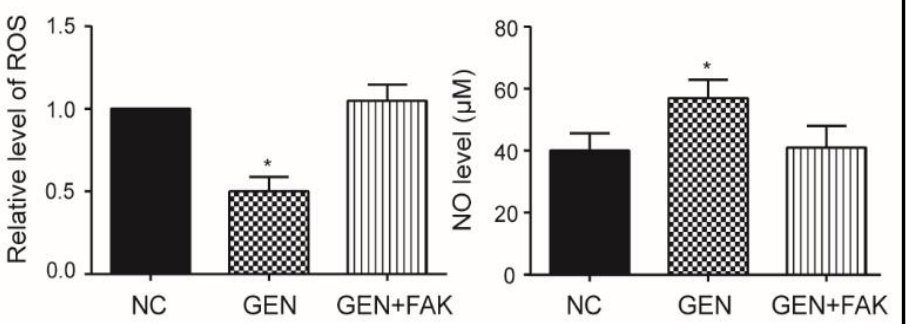

G
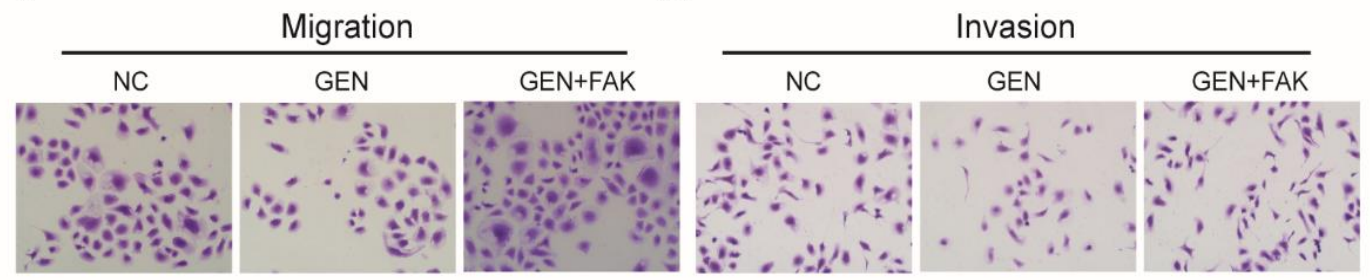

$\mathrm{H}$

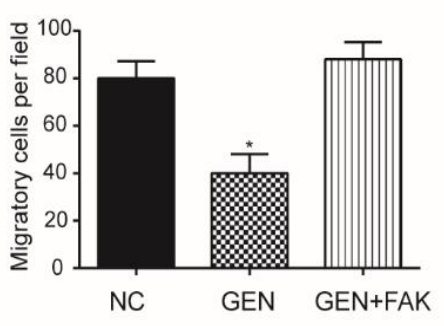

J

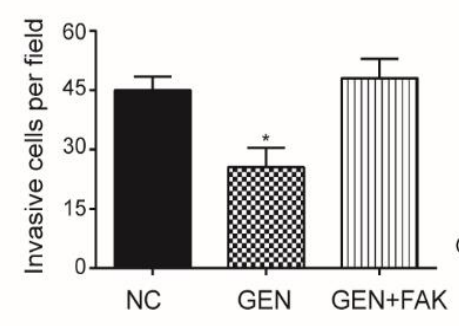

$\mathrm{K}$

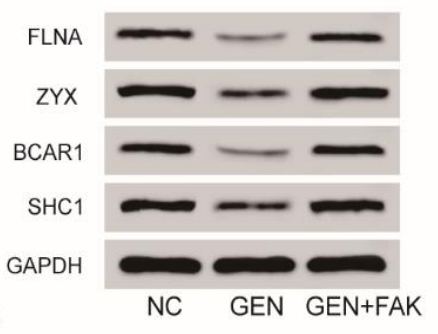

Fig. 8. Genistein reduced the FAK expression level and alleviated the damage to the HU-ESTs. HU-ESTs with different treatment paradigms. Cells in the GEN group were treated with genistein, cells in GEN+FAK group were treated with genistein and overexpressed FAK. (A) qRT-PCR was used to test the relative expression levels of FAK. (B) The relative protein expression of FAK was detected by western blot. (C) MTT examined the cell viability. (D) ROS test was used to detect relative levels of ROS. (E) NO test was used to detect NO level. (F) Transwell experiments were conducted to detect the migratory ability. (G) Transwell experiments were conducted to detect the invasive ability. $(\mathrm{H})$ The histogram shows the number of migrating cells. (J) The histogram shows the number of invading cells. $(\mathrm{K})$ The western blot experiment examined protein levels in the focal adhesion pathway. ${ }^{*} \mathrm{P}<0.05$ indicated statistical significance compared with the NC group. 


\section{Cellular Physiology Cell Physiol Biochem 2018;49:2277-2292 and Biochemistry \begin{tabular}{c|c} 
DOI: 10.1159/000493830 \\
Published online; 27 september 2018
\end{tabular} $\begin{aligned} & \text { O } 2018 \text { The Author(s). Published by S. Karger AG, Basel } \\
& \text { www.karger.com/cpb }\end{aligned}$ \\ Liu et al.: Genistein Inhibited Estradiol-Induced Vascular Endothelial Cell Injury}

\section{Discussion}

In this study, we performed microarray analysis to screen the differential expression gene of FAK. Next, we verified FAK was upregulated in the HU-ESTs, and cell viability and NO levels were significantly weakened, while ROS levels were enhanced in the estradioltreated HUVECs. GO analysis and KEGG analysis of the differentially expressed genes were conducted to screen for functions of cells and pathways. We found that FAK was closely related with the focal adhesion pathway. MTT, ROS and NO detection showed that when FAK was overexpressed, cell viability decreased. Likewise, the transwell experimental results showed that the overexpression of FAK not only promoted the migration and invasion of HUESTs but also activated the adhesion pathway. Next, we found that genistein decreased the expression of $F A K$, indirectly inhibited the activation of the adhesion pathway, and ultimately reduced the estradiol-induced injury of vascular endothelial cells.

Vascular endothelial injury is the first step in atherosclerosis. Long-term cardiovascular disease can cause vascular endothelial cell injury to varying degrees, which is bound to aggravate heart disease [11]. In recent years, there have been more studies on estradiol treatment in endothelial cells. It was reported that estradiol enhanced the recovery of myocardial infarction by increasing the incorporation of bone marrow derivatives [12]. In this study, we found that estradiol-treated endothelial cells might produce large amounts of reactive oxygen species, which caused damage to vascular endothelial cells. Previous experiments have shown that ROS overproduction damages vascular endothelial cells [13], as evidenced by the MTT and NO experiments.

According to previous research, $F A K$ was a key regulator of adhesion renewal, and the precise mechanism of focal adhesion signal pathway activation had been elusive for years [14]. Studies revealed that focal adhesion kinase (FAK) played a vital role in the adhesion of vertebrate cells [15]. Angel Matias Sanchez et al. revealed that FAK might control cell migration, especially in the case of metastasis. FAK overexpression activated the focal adhesion pathway and was associated with the metastatic behavior of breast cancer [16]. The study of Chia-Lung Tsai et al. showed that the overexpression of FAK could stimulate the endometrial cancer cells induced by estradiol and promote adhesion [17]. Similarly, our study investigated the role of high expression of FAK in HU-ESTs. Meanwhile, overexpression of $F A K$ could facilitate the migratory and invasive abilities of endothelial cells induced by estradiol.

The exact mechanism of genistein's vascular protective ability remains a mystery [13]. However, studies have shown that genistein, as a new drug, could protect tumor tissues from the inflammatory reactions and dysfunction caused by TNF- $\alpha$ [18]. In recent years, there have been many studies that confirmed the positive role of genistein in the treatment of diseases. For example, Wei-Fang Li et al. believed that genistein could improve the renal damage induced by ischemia/reperfusion by means of SIRT1 dependence [19]. Gloria GutiérrezVenegas et al. revealed that genistein was able to hinder the LPS-induced inflammatory response in H9c2 cells and decrease damage in myocardial cells due to LPS exposure [20]. Surico D et al. reported that genistein and $17 \beta$-estradiol counteract the effects of peroxidation by an intracellular signal related to Akt and p38 MAPK and through the involvement of ERs and GPER [21]. In addition, Catmull $S$ et al. proposed that dietary genistein rescued reduced basal chloride in the diabetic Jejunum via sex-dependent mechanisms [22]. Likewise, in our study, we found that genistein could reduce the expression of $F A K$, indirectly hindering the activation of the focal adhesion pathway and thereby reducing the damage to estradioltreated endothelial cells.

Inevitably, there are some limitations in the current study. For example, in the study of cell functions, we lacked analysis of cell proliferation and apoptosis. Moreover, in vivo experiments would strengthen our experimental results. 


\section{Cellular Physiology Cell Physiol Biochem 2018;49:2277-2292 \begin{tabular}{l|l|l|l|l} 
DOI: 10.1159/000493830 & $\begin{array}{l}\text { C) } 2018 \text { The Author(s). Published by S. Karger AG, Basel } \\
\text { www.karger.com/cpb }\end{array}$
\end{tabular}}

Liu et al.: Genistein Inhibited Estradiol-Induced Vascular Endothelial Cell Injury

\section{Conclusion}

In summary, FAK reduced the activity of estradiol-treated endothelial cells and promoted their ability to migrate and invade. At the same time, $F A K$ also activated the focal adhesion pathway, which aggravated the damage of vascular endothelial cells. In addition, genistein could reduce the expression level of $F A K$ and indirectly affect the focal adhesion pathway, thereby reduced the damage. Our research showed that genistein might be an important regulatory factor in estradiol-induced endothelial dysfunction.

\section{Acknowledgements}

This study was supported by the Study on the Damage of Vascular Endothelial Cells Induced by Hyperuricemia in Qingdao Municipal Fund (No. 2538); Endovascular Angioplasty Combined with Catheter Thrombolysis for Lower Extremity Deep Venous Thrombosis Combined with Pulmonary Embolism by 2016 Youth Fund of the Affiliated Hospital of Qingdao University (No.2616); "Clinical +X" Project Fund of Qingdao University (2017M24).

\section{Disclosure Statement}

The authors confirm that they have no potential conflict of interest.

\section{References}

1 Matsumoto A, Pasut A, Matsumoto M, Yamashita R, Fung J, Monteleone E, Saghatelian A, Nakayama KI, Clohessy JG, Pandolfi PP: mTORC1 and muscle regeneration are regulated by the LINC00961-encoded SPAR polypeptide. Nature 2017;541:228-232.

2 Li Y, Zhang X, Yang W, Li C, Chu Y, Jiang H, Shen Z: Mechanism of the protective effects of the combined treatment with rhynchophylla total alkaloids and sinapine thiocyanate against a prothrombotic state caused by vascular endothelial cell inflammatory damage. Exp Ther Med 2017;13:3081-3088.

-3 Chen CH, Chen TH, Wu MY, Chou TC, Chen JR, Wei MJ, Lee SL, Hong LY, Zheng CM, Chiu IJ, Lin YF, Hsu CM, Hsu YH: Far-infrared protects vascular endothelial cells from advanced glycation end products-induced injury via PLZF-mediated autophagy in diabetic mice. Sci Rep 2017;7:40442.

4 Amiri Gheshlaghi S, Mohammad Jafari R, Algazo M, Rahimi N, Alshaib H, Dehpour AR: Genistein modulation of seizure: involvement of estrogen and serotonin receptors. J Nat Med 2017;71:537-544.

5 Banerjee S, Li Y, Wang Z, Sarkar FH: Multi-targeted therapy of cancer by genistein. Cancer Lett 2008;269:226-242.

6 Du DS, Ma XB, Zhang JF, Zhou XY, Li Y, Zhang YM, Qiao WL: The protective effect of capsaicin receptormediated genistein postconditioning on gastric ischemia-reperfusion injury in rats. Dig Dis Sci 2010;55:3070-3077.

7 Lee SH, Lee JH, Asahara T, Kim YS, Jeong HC, Ahn Y, Jung JS, Kwon SM: Genistein promotes endothelial colony-forming cell (ECFC) bioactivities and cardiac regeneration in myocardial infarction. PLoS One 2014;9:e96155.

-8 Wang Q, Wang Y, Fritz D, Rajshankar D, Downey GP, McCulloch CA: Interactions of the protein-tyrosine phosphatase-alpha with the focal adhesion targeting domain of focal adhesion kinase are involved in interleukin-1 signaling in fibroblasts. J Biol Chem 2014;289:18427-18441.

-9 Zhang J, Hochwald SN: The role of FAK in tumor metabolism and therapy. Pharmacol Ther 2014;142:154163.

10 Sulzmaier FJ, Jean C, Schlaepfer DD: FAK in cancer: mechanistic findings and clinical applications. Nat Rev Cancer 2014;14:598-610.

11 Ma S, Yao S, Tian H, Jiao P, Yang N, Zhu P, Qin S: Pigment epithelium-derived factor alleviates endothelial injury by inhibiting Wnt/beta-catenin pathway. Lipids Health Dis 2017;16:31. 


\section{Cellular Physiology Cell Physiol Biochem 2018;49:2277-2292

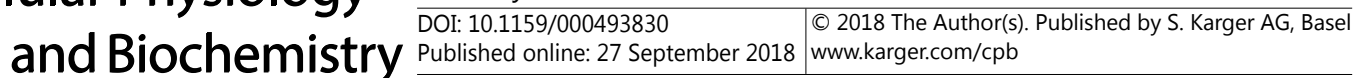

Liu et al.: Genistein Inhibited Estradiol-Induced Vascular Endothelial Cell Injury

12 Iwakura A, Shastry S, Luedemann C, Hamada H, Kawamoto A, Kishore R, Zhu Y, Qin G, Silver M, Thorne T, Eaton L, Masuda H, Asahara T, Losordo DW: Estradiol enhances recovery after myocardial infarction by augmenting incorporation of bone marrow-derived endothelial progenitor cells into sites of ischemia-induced neovascularization via endothelial nitric oxide synthase-mediated activation of matrix metalloproteinase-9. Circulation 2006;113:1605-1614.

13 Han S, Wu H, Li W, Gao P: Protective effects of genistein in homocysteine-induced endothelial cell inflammatory injury. Mol Cell Biochem 2015;403:43-49.

14 Sanchez AM, Flamini MI, Zullino S, Gopal S, Genazzani AR, Simoncini T: Estrogen receptor-\{alpha\} promotes endothelial cell motility through focal adhesion kinase. Mol Hum Reprod 2011;17:219-226.

15 Fujimoto J, Sawamoto K, Okabe M, Takagi Y, Tezuka T, Yoshikawa S, Ryo H, Okano H, Yamamoto T: Cloning and characterization of Dfak56, a homolog of focal adhesion kinase, in Drosophila melanogaster. J Biol Chem 1999;274:29196-29201.

16 Sanchez AM, Flamini MI, Baldacci C, Goglia L, Genazzani AR, Simoncini T: Estrogen receptor-alpha promotes breast cancer cell motility and invasion via focal adhesion kinase and N-WASP. Mol Endocrinol 2010;24:2114-2125.

17 Tsai CL, Wu HM, Lin CY, Lin YJ, Chao A, Wang TH, Hsueh S, Lai CH, Wang HS: Estradiol and tamoxifen induce cell migration through GPR30 and activation of focal adhesion kinase (FAK) in endometrial cancers with low or without nuclear estrogen receptor alpha (ERalpha). PLoS One 2013;8:e72999.

18 Jia Z, Babu PV, Si H, Nallasamy P, Zhu H, Zhen W, Misra HP, Li Y, Liu D: Genistein inhibits TNF-alpha-induced endothelial inflammation through the protein kinase pathway A and improves vascular inflammation in C57BL/6 mice. Int J Cardiol 2013;168:2637-2645.

19 Li WF, Yang K, Zhu P, Zhao HQ Song YH, Liu KC, Huang WF: Genistein Ameliorates Ischemia/ReperfusionInduced Renal Injury in a SIRT1-Dependent Manner. Nutrients 2017;9.

-20 Gutierrez-Venegas G, Torras-Ceballos A, Gomez-Mora JA, Fernandez-Rojas B: Luteolin, quercetin, genistein and quercetagetin inhibit the effects of lipopolysaccharide obtained from Porphyromonas gingivalis in H9c2 cardiomyoblasts. Cell Mol Biol Lett 2017;22:19.

-21 Surico D, Ercoli A, Farruggio S, Raina G, Filippini D, Mary D, Minisini R, Surico N, Pirisi M, Grossini E: Modulation of Oxidative Stress by 17 beta-Estradiol and Genistein in Human Hepatic Cell Lines In vitro. Cell Physiol Biochem 2017;42:1051-1062.

22 Catmull S, Masood F, Schacht S, Dolan R, Stegman D, Leung L, Al-Nakkash L: Dietary Genistein Rescues Reduced Basal Chloride Secretion in Diabetic Jejunum via Sex-Dependent Mechanisms. Cell Physiol Biochem 2016;40:335-346. 